\title{
Thermal diffusion in nanostructured porous InP
}

\author{
R SRINIVASAN* and K RAMACHANDRAN ${ }^{\dagger}$ \\ Department of Physics, Thiagarajar College, Madurai 625 009, India \\ ${ }^{\dagger}$ School of Physics, Madurai Kamaraj University, Madurai 625 021, India
}

MS received 19 March 2008; revised 14 June 2008

\begin{abstract}
Nanostructured porous InP samples were prepared by electrochemical anodic dissolution of InP for various current densities and etching periods. The samples were characterized by SEM and photoluminescence (PL) where a blue shift was observed in PL. Thermal properties studied by photoacoustic (PA) spectroscopy revealed one order decrease in thermal conductivity of porous InP compared to the bulk. Further it is shown that the thermal conductivity of porous InP decreases with decrease in size of the particles.
\end{abstract}

Keywords. Porous InP; photoacoustics; thermal conductivity; phonon confinement; quantum confinement.

\section{Introduction}

Porous silicon has opened up new research fields for investigating porous layers in other semiconducting materials (Canham 1990). Many reports on the compound semiconductors, GaP (Anedda et al 1995; Marin et al 1996), SiGe (Ksendzov et al 1993), SiC (Shor et al 1993) and GaAs (Cullis et al 1997) in the form of porous layers are already available in literature. Porous InP was studied extensively for the optical properties by Liu and Duan (2001) under various conditions, as InP is a very useful material in light-emitting diodes and solar cells.

Eventhough the surface morphology and optical properties of porous InP (Liu 2001; Liu and Duan 2001) were studied by using SEM and PL measurements, respectively by several researchers, only very few works are available on porous InP for various current densities and etching times. Similarly works on thermal diffusion in porous InP are rarely reported. Here we report the results of optical and thermal properties of porous InP for different sizes of the nanocrystallites.

\section{Experimental}

\subsection{Preparation of porous $\operatorname{InP}(P I P)$}

The porous layers on InP surface were prepared by electrochemical anodic dissolution of InP (100) in 48\% hydrofluoric acid, $\mathrm{H}_{2} \mathrm{O}$ and ethanol with platinum electrode as cathode. This InP single crystal was grown by Czochralski (CZ) method which was then cleaved in (100) as wafers of thickness, $1060 \mu \mathrm{m}$. The electrolyte was prepared by mixing $\mathrm{HF}(48 \%), \mathrm{H}_{2} \mathrm{O}$ and ethanol in $1: 1: 2$ ratio. The ele-

*Author for correspondence (r_srini2067@yahoo.co.in) ctrolyte to be used in the preparation of porous InP was discussed in detail by Schmuki et al (2000), where $\mathrm{HCl}$, $\mathrm{HBr}$ and $\mathrm{HF}$ solutions were used for the dissolution process of InP. It was proved that uptake of electrolyte anions was the highest for $\mathrm{HBr}$ and lowest for $\mathrm{HF}$ and the finest pore was obtained in the dark. Still this electrolyte HF was used here, as Liu and Duan (2001) had carried out all his studies only with $\mathrm{HF}$ and also in HF, porosity can be controlled as the uptake of anions is low. The porous layers on the surface of these samples were prepared for (i) different current densities $\left(10,30,40\right.$ and $\left.60 \mathrm{~mA} / \mathrm{cm}^{2}\right)$ at constant etching time of $15 \mathrm{~min}$ and (ii) different etching times $(5,15$ and $30 \mathrm{~min})$ at a constant current density of $30 \mathrm{~mA} / \mathrm{cm}^{2}$ under dark condition. For convenience of discussions, these samples are identified as given in table 1 [for example InP etched at a current density of $10 \mathrm{~mA}$ / $\mathrm{cm}^{2}$ (from a constant current source) for etching time $5 \mathrm{~min}$ is identified as sample 1 and so on].

\subsection{Characterization of porous InP (PIP)}

The scanning electron microscopy (SEM) was used to investigate the etched InP samples using Philips XL30 ESEM to confirm the porosity. Only some typical SEM pictures of PIP (samples 1, 2 and 6) are shown here in figures 1(a), (b) and (c) and porosity of 26, 35 and $45 \%$ is observed for these samples. Here the porosity $(\mathrm{P})$ of the sample is determined by weighing the InP substrate both before $\left(m_{1}\right)$ and after $\left(m_{2}\right)$ the etching process and again after porous layer $\left(m_{3}\right)$ has been removed. The porosity is given by (Wolf and Brendel 2006)

$$
P=\left(m_{1}-m_{2}\right) /\left(m_{1}-m_{3}\right) .
$$

The compositions of these porous samples are analysed with energy dispersive X-rays (EDAX) for the atomic 
percentage of In and $\mathrm{P}$ in InP (figure 2) which are 53.7 and $47.3 \%$, respectively. This shows that no other impurities with significant percentage of concentration entered into the PIP samples because of anodic etching.

\subsection{Photoluminescence}

Photoluminescence (PL) emission spectra of PIP samples 1-6 are recorded at room temperature using a spectroflurophotometer (Shimadzu RF-5000). Figure 3 shows spectra of PIP samples (5 and 2) prepared at different etching times and figure 4 shows spectra of samples (2 and 4) prepared at different current densities.

The change in bandgap, $\Delta E$, due to size quantization was given by Brus (1994) in effective mass approximation as

$$
\Delta E=\frac{h^{2}}{2 d^{2}}\left(\frac{1}{m_{e}^{*}}+\frac{1}{m_{\mathrm{h}}^{*}}\right) .
$$

The depth of potential well for these nanocrystallites was given by $\Delta E=E_{\max }-E_{\mathrm{g}}$, where $E_{\max }$ is the PL peak energy and $E_{\mathrm{g}}$ the energy gap of bulk InP, $d$ the diameter of the spherical particle, and $m_{\mathrm{e}}^{*}$ and $m_{\mathrm{h}}^{*}$ are the electron and hole effective masses, respectively. At $300 \mathrm{~K}, E_{\mathrm{g}}=$ $1.34 \mathrm{eV}, m_{\mathrm{e}}^{*}=0.073$ and $m_{\mathrm{h}}^{*}=0.4$ (Madelung 1991). The PL peak energies and calculated particle size of PIP samples from (2) are shown in table 2. The observed PL was due to InP nanocrystallites of about 6-9 $\mathrm{nm}$ in diameter.

Samples 1-4 and 5, 2 and 6 (table 2) show that the size of particles varies with current density and etching period, respectively. The theoretical calculations by Brus (1994) based on a hyperbolic potential could be used to find the size of the particles when they are above $2 \mathrm{~nm}$ and this is the limit for this approximation. Eventhough here XRD measurements are not done, from our experience on various nano systems, where Debye Scherrer formula was used for the same, we find the agreement with XRD is within $2 \%$ and so photoluminescence can be used to find out size of the particles using the Brus formula.

The variation in particle size with increase in current density is appreciable compared to etching period. For

Table 1. Etching parameters and sample identification.

\begin{tabular}{lcc}
\hline & \multicolumn{2}{c}{ Etching parameters } \\
\cline { 2 - 3 } $\begin{array}{l}\text { Sample } \\
\text { InP) }\end{array}$ & $\begin{array}{c}\text { Current density } \\
\left(\mathrm{mA} / \mathrm{cm}^{2}\right)\end{array}$ & $\begin{array}{c}\text { Etching time } \\
(\mathrm{min})\end{array}$ \\
\hline Sample 1 & 10 & 15 \\
Sample 2 & 30 & 15 \\
Sample 3 & 40 & 15 \\
Sample 4 & 60 & 15 \\
Sample 5 & 30 & 5 \\
Sample 6 & 30 & 30 \\
\hline
\end{tabular}

example, the particle size decreases from 7.0 to $6.4 \mathrm{~nm}$ as the etching period increases from 5 to $30 \mathrm{~min}$ and 8.5 to $6 \cdot 2 \mathrm{~nm}$ as the current density increases from 30 to $60 \mathrm{~mA} /$ $\mathrm{cm}^{2}$. This change in particle size causes appreciable blue shift in peak position of the PL. The blue shift in the
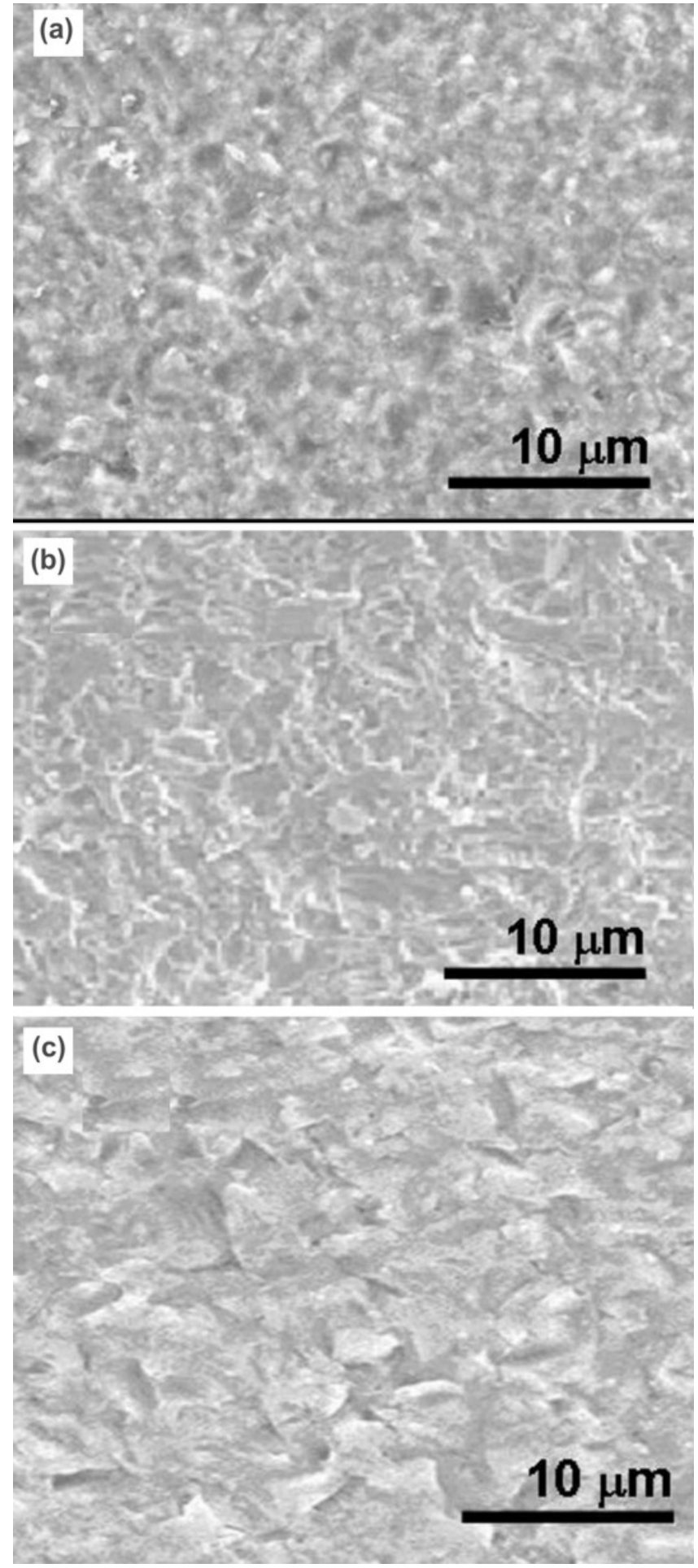

Figure 1. SEM pictures of (a) sample 1, (b) sample 2 and (c) sample 6. 
peak position is due to quantum confinement (Lockwood et al 1999).

The PL emission peaks of all samples (table 2) are in the range of $600-750 \mathrm{~nm}$. The PL peak positions observed in our experiment is consistent with the reported results (Chernoutsan et al 2000; Schmuki et al 2000). Figures 3 and 4 and table 2 also show that peak positions (corresponding peak energies) in all porous samples are blue shifted from the bulk InP whose PL peak is at $1.34 \mathrm{eV}$ (Levinshtein et al 1996). The most consistent explanation for the observed spectral effect (blue shift) is quantum confinement. In a quantum confinement emission mechanism, the PL peak is shifted to higher energy compared with bulk InP in proportion to the degree of electron confinement. This is consistent with the theory of porous Si for increasing porosity (Dalba et al 1998a, b). The porosity observed in samples 1 and 2 (figures 1(a) and (b)) are 26 and $35 \%$ and in samples 2 and 6 (figures 1(b) and (c)), they are 35 and $45 \%$ i.e. the porosity increases with increase in current density and etching period. These different porosities result in different nanometer sized InP crystallites. Further, the other observed peak i.e. $608 \mathrm{~nm}$ in sample 5 other than major peak $(675 \mathrm{~nm})$ imply that there exists a shoulder peak arising due to the different nanometer sized crystallites and non-uniformity in the porosity.

On comparing the samples 5,2 and 6, there is a relative blue shift in PL peak energy with increasing etching time, for example, a relative blue shift of $11 \mathrm{~nm}$ is observed between samples 5 and 2 (table 2) and similar trend is observed for samples 2 and 6 . Whereas on increasing the current density, the relative shift is little higher among the samples, i.e. an appreciable blue shift is observed for increasing current density than the etching period.

Generally the bulk (direct bandgap) semiconductors show PL emission corresponding to their bandgap ener-

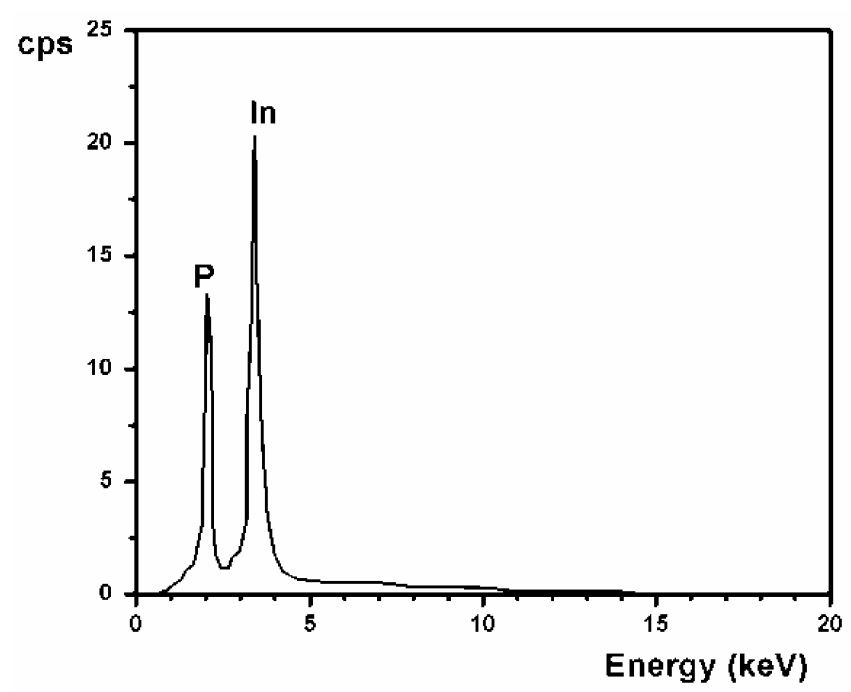

Figure 2. EDAX picture of porous InP. gies which is $1.34 \mathrm{eV}$ in case of InP (Liu 2001). When porous layers are formed by electrochemical etching process, pores are formed on the surface of the bulk materials and these formations of pores can be controlled by current density and etching period. The porosity of these samples can be calculated by mass loss during etching as discussed earlier. As the current density and etching period is increased the porosity of the sample also increases due to more etching (Shen and Toyoda 2003). Further, during the etching process, InP nanocrystallites are formed on the surface and particle size of these nanocrystallites decreases with increase in porosity. This decrease in particle size, due to increase in porosity, is responsible for blue shift in the bandgap energies. This is evident even from (2) given here.

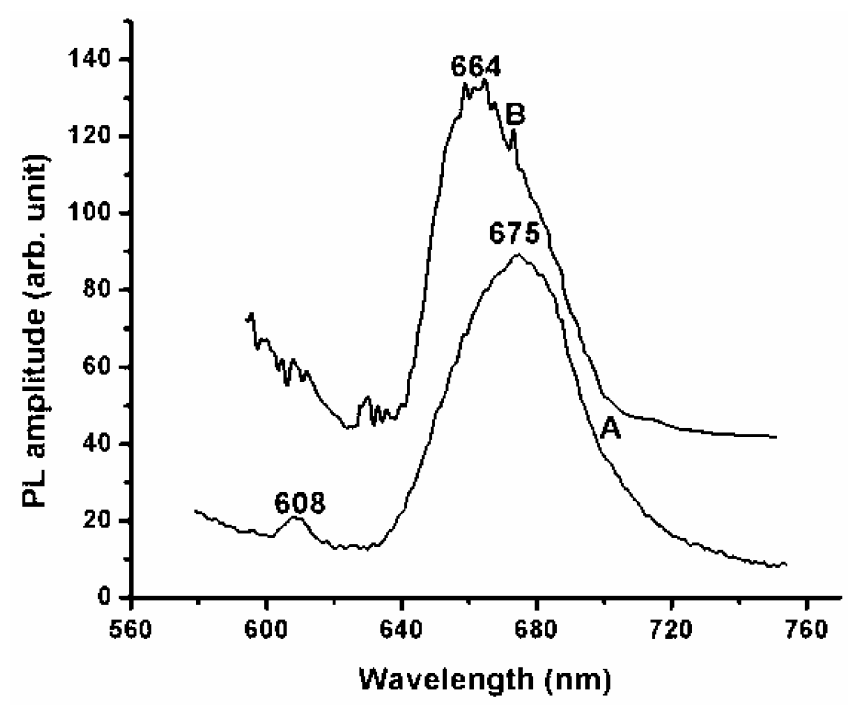

Figure 3. PL emission spectra of porous InP for etching current density $30 \mathrm{~mA} / \mathrm{cm}^{2}$; A. sample 5 and B. sample 2 .

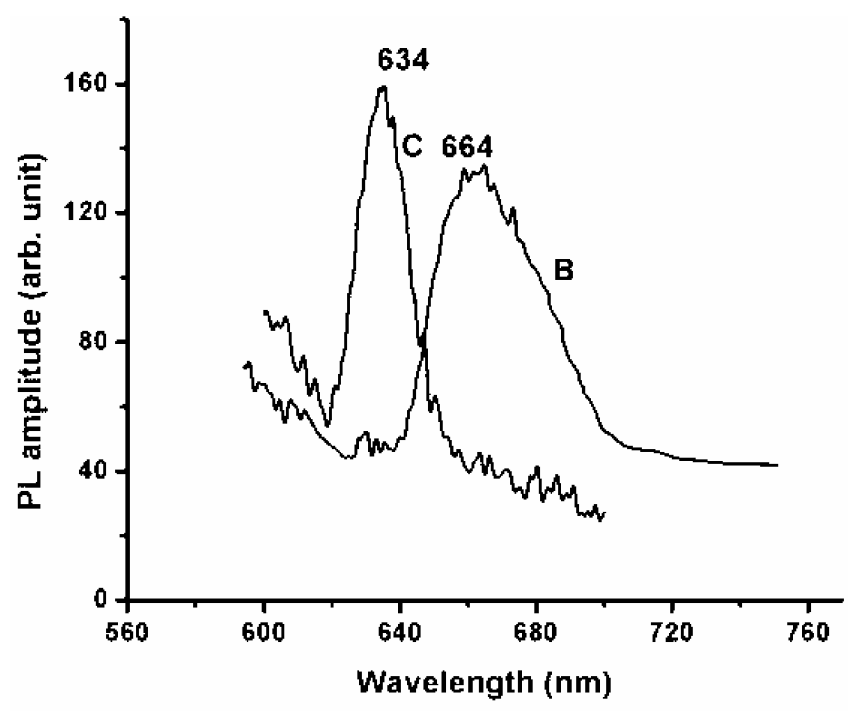

Figure 4. PL emission spectra of porous $\mathrm{InP}$ for an etching time of 15 min: B. sample 2 and $\mathbf{C}$. sample 4. 
When the etching current is increased, porosity would increase, but when the current is kept constant and time is increased, one would not expect much porosity, as at one particular instant of time, the porosity would have reached its limit and so further increase in time will not have any effect. The dependence of the PL spectra of porous GaAs as a function of current density by Beji et al (2006) reported a yellow band undergoing a blue shift on increasing the current density, which is consistent with our present study.

\subsection{Thermal properties of porous InP}

Porous InP, prepared for various current densities and etching periods are now used to study the thermal properties as reports on thermal diffusion in PIP are not available in literature. Here the thermal properties of PIP are studied using photoacoustic (PA) technique, which involves direct measurement of the energy absorbed by a material as a result of its interaction with the photon beam. In the present set up, polychromatic light from a xenon lamp (400 W, Jobin Yvon) is mechanically chopped by a mechanical chopper (Model number PAR 650) and focused onto the sample. The sample is placed in a PA cell and a microphone is placed very near to the sample. The PA signal from the sample is fed to a lock-in amplifier (Model Perkin Elmer 7225 DSP) and PA signal amplitude $(S)$ is recorded as a function of chopping frequency $(f)$. The experimental technique and method of calculations are already discussed in our earlier paper (Srinivasan et al 2007).

The thermal diffusivity $(\alpha)$ was determined from the thermal diffusion model for the photoacoustic effect, which states that, for an optically opaque sample, the pressure fluctuations are given by (Rosencwaig 1980)

$$
\delta p=\gamma p_{0} I_{0}\left(\alpha_{\mathrm{g}} \alpha_{\mathrm{s}}\right)^{1 / 2} \exp j\left(\omega t-\frac{\pi}{2}\right) / 2 \pi T_{0} l_{\mathrm{g}} \kappa_{\mathrm{s}} f \sin h\left(l_{\mathrm{s}} \sigma_{\mathrm{s}}\right),
$$

where $\gamma$ is the air specific heat ratio, $p_{0}$ the ambient pressure, $I_{0}$ the incident light beam intensity, $T_{0}$ the room temperature, $f$ the chopping frequency and $l_{\mathrm{i}}, k_{\mathrm{i}}$, and $\alpha_{i}$ are the thickness, thermal conductivity and thermal diffusivity of material $i$, respectively.

Table 2. PL peak energy and particle size (eqn. (2)) of porous $\mathrm{InP}$ (error $\sim \pm 1 \%$ ).

\begin{tabular}{lccc}
\hline $\begin{array}{l}\text { Sample } \\
\text { (InP) }\end{array}$ & $\begin{array}{c}\text { PL peak } \\
(\mathrm{nm})\end{array}$ & $\begin{array}{c}\text { PL peak energy } \\
(\mathrm{eV})\end{array}$ & $\begin{array}{c}\text { Particle size } \\
(\mathrm{nm})\end{array}$ \\
\hline Sample 1 & 735 & $1 \cdot 68$ & $8 \cdot 5$ \\
Sample 2 & 664 & $1 \cdot 87$ & $6 \cdot 8$ \\
Sample 3 & 645 & 1.92 & $6 \cdot 5$ \\
Sample 4 & 634 & 1.96 & $6 \cdot 2$ \\
Sample 5 & 675 & $1 \cdot 83$ & $7 \cdot 0$ \\
Sample 6 & 640 & 1.94 & $6 \cdot 4$ \\
\hline
\end{tabular}

The subscript $i$ denotes either sample (s) or gas (g) regions and $\sigma_{i}=(1+j) a_{i}$ with

$$
a_{i}=\left(\frac{\pi f}{\alpha_{i}}\right)^{1 / 2},
$$

the complex thermal diffusion coefficient of material ' $i$ '. Particularly, for an optically opaque and thermally thick sample $\left(l_{\mathrm{s}} \sigma_{\mathrm{s}}>>1\right)$, the expression for the photoacoustic amplitude is given by

$$
S=\frac{A}{f} \exp \left(-a f^{1 / 2}\right)
$$

where the constant $A$, apart from geometric constants, include factors such as the light intensity, temperature, gas thermal properties and

$$
a=\left(\frac{\pi l_{\mathrm{s}}^{2}}{\alpha_{\mathrm{s}}}\right)^{1 / 2} \text {. }
$$

From the slope of the graph (figure 5) connecting $\ln (f \cdot S)$ and $\sqrt{f}$ (where $S$ is the strength of the PA signal), thermal diffusivity $(\alpha)$ of the sample is deduced. The thermal diffusivity of porous sample is calculated using the relation

$$
a=\left(\frac{\pi l^{2}}{\alpha}\right)^{1 / 2}
$$

where $\alpha$ is the thermal diffusivity, $a$ the slope of the graph connecting $\ln (f \cdot S)$ and $\sqrt{f}$, and $l$ the thickness of the sample. Thermal conductivity $(k)$ is then calculated using the relation

$$
\kappa=\alpha \rho C_{\mathrm{p}} \text { in units of } \mathrm{W} / \mathrm{m}-\mathrm{K},
$$

where $\rho$ is the density and $C_{\mathrm{p}}$ the specific heat capacity of bulk InP. The measured values of thermal diffusivity and conductivity are given in table 3 .

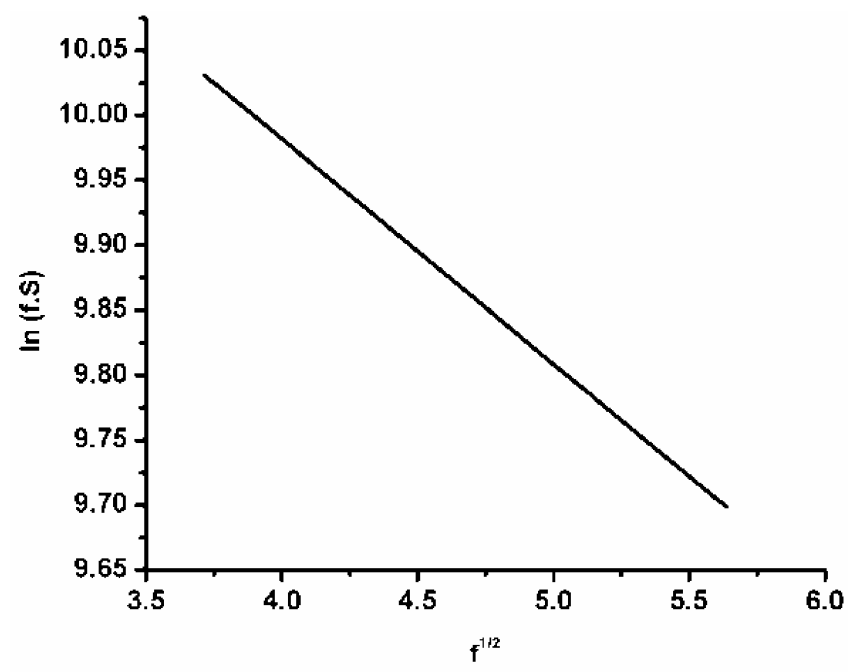

Figure 5. Variation of PA signal with chopping frequency for porous InP (sample 2). 
Table 3. Thermal diffusivity and conductivity of porous $\mathrm{InP}$ at room temperature (error $\sim \pm 1 \%$ )

\begin{tabular}{lcccc}
\hline $\begin{array}{l}\text { Sample } \\
\text { InP }\end{array}$ & $\begin{array}{c}\text { Porosity } \\
(\%)\end{array}$ & $\begin{array}{c}\text { Particle size } \\
(\mathrm{nm})\end{array}$ & $\begin{array}{c}\text { Thermal diffusivity } \\
\times 10^{-5}\left(\mathrm{~m}^{2} / \mathrm{s}\right)\end{array}$ & $\begin{array}{c}\text { Thermal conductivity } \\
\left(\mathrm{Wm}^{-1} \mathrm{~K}^{-1}\right)\end{array}$ \\
\hline Sample 1 & 26 & $8 \cdot 5$ & $0 \cdot 26$ & $3 \cdot 9$ \\
Sample 2 & 35 & $6 \cdot 8$ & $0 \cdot 19$ & $2 \cdot 8$ \\
Sample 3 & 42 & $6 \cdot 5$ & $0 \cdot 18$ & $2 \cdot 6$ \\
Sample 4 & 55 & $6 \cdot 2$ & $0 \cdot 12$ & $1 \cdot 6$ \\
Sample 5 & 30 & $7 \cdot 0$ & $0 \cdot 22$ & $3 \cdot 3$ \\
Sample 6 & 45 & $6 \cdot 4$ & $0 \cdot 17$ & $2 \cdot 5$ \\
Bulk* & - & - & $3 \cdot 72$ & $68 \cdot 0$ \\
\hline
\end{tabular}

${ }^{*}$ www.ioffe.ru/SVA/NSM/Semicond/InP/thermal.html

It is observed that (table 3 ) the thermal diffusivities and conductivities of these samples are atleast one order less than the bulk InP. This decrease is due to the decrease in mean free path resulting from multiple scattering of the phonons. Further the dangling bonds of porous InP occurring at the planar InP can lead to electron-hole recombinations, for which part of the thermal energy is used and the trapped carrier acquiring a thermal velocity,

$$
\sqrt{\frac{8 k T}{\pi m^{*}}},
$$

where $m^{*}$, the effective mass of the trapped carrier, from this energy. This also leads to the multiple scattering and hence a reduction in thermal conductivity.

It is observed that there is decrease in thermal conductivity (table 3) as the current density increases; for example, the thermal conductivity decreases from 3.9 to $1.6 \mathrm{Wm}^{-1} \mathrm{~K}^{-1}$ as the current density increases from 10 to $60 \mathrm{~mA} / \mathrm{cm}^{2}$ and this is due to variation in particle size $(\sim 2.2 \mathrm{~nm})$ and porosity (26 and $55 \%$ ). A similar behaviour is observed for increase in etching period also. This is due to the phonon confinement around nano-crystallites formed due to the decrease in particle size. Thus the reduction in thermal conductivity is because of decrease in phonon mean free path due to phonon confinement around nanocrystallites. That is, when the phonon mean free path is less than the crystallite size, phonons are confined and so the thermal conductivity is reduced.

Shen and Toyoda (2003) reported that the thermal conductivity of PS films decreases with increase in porosity for porous silicon (PS), by photoacoustic technique. Zou and Balandin (2001) investigated phonon heat conduction in a semiconductor nanowire and reported that phonon confinement and boundary scattering lead to a significant decrease of the lattice thermal conductivity, which is consistent with our present results.

\section{Conclusions}

Porous layers on InP are prepared for different current densities and etching periods. An appreciable change in porosity and particle size was observed with current density and etching period as well. The observed blue shift in PL peak is explained by using quantum confinement. Similarly the decrease in thermal conductivity of porous InP is due to phonon confinement. Since reports on thermal properties of porous InP are very sparse, the present values add new data to literature, as this material (bandgap widening and lower thermal conductivity) is suitable for optoelectronic devices.

\section{Acknowledgements}

The authors acknowledge the University Grants Commission (UGC), India, for financial support in the form of a minor research project (UGC Link No. 2386). Also the authors thank Prof. D B Sirdeshmukh, Kakatiya University, Warangal, for providing the bulk InP samples.

\section{References}

Anedda A, Serpl A, Karavanskii V K and Lehizli V M 1995 Appl. Phys. Lett. 673316

Beji L, Missaoui A, Fouzri A, Ben Quada H, Maaref H and Bouazizi A 2006 Microelectronic Journal 37783

Brus L E 1994 J. Chem. Phys. 804403

Canham L T 1990 Appl. Phys. Lett. 571046

Chernoutsan K, Dneprovskii V, Shaligina O and Zhukov E 2000 Phys. Status Solidi (a) 182347

Cullis A G, Canham L T and Calcott P D G 1997 J. Appl. Phys. 82909

Dalba G, Daldosso N, Fornasini P, Gariziola R, Grisenti R and Rocca F 1998a J. Non-Cryst. Solids 232-234 370

Dalba G, Daldosso N, Diop D, Fornasini P, Grisenti R and Rocca F 1998b J. Lumin. 80103

Ksendzov A, Fahauser R W, George T, Pike W T, Vasquez R P and Tylor A P 1993 Appl. Phys. Lett. 62200

Levinshtein M, Rumyantsev S and Shur M 1996 Schmidt handbook series on semiconductor parameters (London: World Scientific), Vol. 1, pp. 169-190

Liu Aimin 2001 Nanotechnology 12 L1

Liu Aimin and Duan Changkui 2001 Solid State Electron. 45 2089

Lockwood D J, Schmuki P, Labbe H J and Fraser J W 1999 Physica E4 102 
Madelung O 1991 Semiconductors: Group IV elements and III-V compounds (Berlin: Springer) p. 101

Marin I, Hamstra M A and Vanmaekelbergh D 1996 J. Electrochem. Soc. 143113

Rosencwaig A 1980 Photoacoustics and photoacoustic spectroscopy (New York: Wiley)

Schmuki P, Santinacci L, Djenizian T and Lockwood D J 2000 Phys. Status Solidi (a) $\mathbf{1 8 2} 51$
Shen Qing and Toyoda Taro 2003 Rev. Sci. Instrum. 74601

Shor S, Grimberg I, Weiss B Z and Kuetz A D 1993 Appl. Phys. Lett. 622836

Srinivasan R, Jayachandran M and Ramachandran K 2007 Cryst. Res. Technol. 42226

Wolf A and Brendel R 2006 Thin Solid Films 513385

Zou Jie and Balandin Alexander 2001 J. Appl. Phys. 89 2932 